University of Wollongong

Research Online

Faculty of Social Sciences - Papers (Archive) Faculty of Arts, Social Sciences \& Humanities

$1-1-2016$

\title{
Schizotypy and auditory mismatch negativity in a non-clinical sample of young adults.
}

Samantha J. Broyd

University of Wollongong, sbroyd@uow.edu.au

Patricia Michie

University of Newcastle

Jason Bruggemann

University of New South Wales, jmb02@uow.edu.au

Hendrika $\mathrm{H}$. van Hell

University of Wollongong, erikavh@uow.edu.au

Lisa-Marie Greenwood

University of Wollongong, Img001@uowmail.edu.au

See next page for additional authors

Follow this and additional works at: https://ro.uow.edu.au/sspapers

Part of the Education Commons, and the Social and Behavioral Sciences Commons

Research Online is the open access institutional repository for the University of Wollongong. For further information contact the UOW Library: research-pubs@uow.edu.au 
Schizotypy and auditory mismatch negativity in a non-clinical sample of young adults.

\author{
Abstract \\ Schizophrenia may be conceptualised using a dimensional approach to examine trait-like expression such \\ as schizotypy within non-clinical populations to better understand pathophysiology. \\ Keywords \\ schizotypy, auditory, mismatch, adults., negativity, non-clinical, sample, young \\ Disciplines \\ Education | Social and Behavioral Sciences

\section{Publication Details} \\ Broyd, S. J., Michie, P., Bruggemann, J., van Hell, H. H., Greenwood, L., Croft, R. J., Todd, J., Lenroot, R. \& \\ Solowij, N. (2016). Schizotypy and auditory mismatch negativity in a non-clinical sample of young adults.. \\ Psychiatry Research: Neuroimaging, 254 83-91.

\section{Authors} \\ Samantha J. Broyd, Patricia Michie, Jason Bruggemann, Hendrika H. van Hell, Lisa-Marie Greenwood, \\ Rodney J. Croft, Juanita Todd, Rhoshel Lenroot, and Nadia Solowij
}


Schizotypy and auditory mismatch negativity in a non-clinical sample of young adults

Samantha J. Broyd ${ }^{a^{*}}$, Patricia T. Michie ${ }^{b}$, Jason Bruggemann ${ }^{c}$, Hendrika H. van Hell ${ }^{a}$, Lisa-marie Greenwood $^{\mathrm{a}}$, Rodney J. Croft ${ }^{\mathrm{a}}$, Juanita Todd ${ }^{\mathrm{b}}$, Rhoshel Lenroot ${ }^{\mathrm{c}}$ and Nadia Solowij ${ }^{\mathrm{a}}$

${ }^{a}$ School of Psychology, Centre for Health Initiatives and Illawarra Health and Medical Research Institute University of Wollongong, Wollongong NSW Australia;

${ }^{\mathrm{b}}$ School of Psychology and Priority Research Centre for Translational Neuroscience and Mental Health, University of Newcastle, Newcastle NSW Australia;

${ }^{\mathrm{c}}$ School of Psychiatry, University of New South Wales and Neuroscience Research Australia, Sydney, Australia.

* Corresponding author: Samantha Broyd, School of Psychology, University of Wollongong NSW Australia; E-mail: sbroyd@uow.edu.au; Tel. +61 24221 4515; Fax. +61 242214163

Word count: Manuscript: 4988 words (limit 5000 words); Abstract: 200 words 


\begin{abstract}
Schizophrenia may be conceptualised using a dimensional approach to examine trait-like expression such as schizotypy within non-clinical populations to better understand pathophysiology. A candidate psychosis-risk marker, the auditory mismatch negativity (MMN) is thought to index the functionality of glutamatergic NMDA receptor mediated neurotransmission. Although the MMN is robustly reduced in patients with schizophrenia, the association between MMN and schizotypy in the general population is under-investigated. Thirty-five healthy participants completed the Schizotypal Personality Questionnaire (SPQ) and a multi-feature MMN paradigm (standards $82 \%, 50 \mathrm{~ms}, 1000 \mathrm{~Hz}, 80 \mathrm{~dB}$ ) with duration $(100 \mathrm{~ms})$, frequency $(1200 \mathrm{~Hz})$ and intensity $(90 \mathrm{~dB})$ deviants (6\% each). Spearman's correlations were used to explore the association between schizotypal personality traits and MMN amplitude. Few associations were identified between schizotypal traits and MMN. Higher Suspiciousness subscale scores tended to be correlated with larger frequency MMN amplitude. A median-split comparison of the sample on Suspiciousness scores showed larger MMN (irrespective of deviant condition) in the High compared to the Low Suspiciousness group. The trend-level association between MMN and Suspiciousness is in contrast to the robustly attenuated MMN amplitude observed in schizophrenia. Reductions in MMN may reflect a schizophrenia-disease state, whereas nonclinical schizotypy may not be subserved by similar neuropathology.
\end{abstract}

Key words: Mismatch negativity; MMN; schizotypy; suspiciousness; schizophrenia; event-related potential; ERP 


\section{Highlights:}

- Investigating schizotypy within non-clinical populations may allow us to better understand the pathophysiology of schizophrenia

- $\mathrm{MMN}$ is robustly attenuated in patients with schizophrenia but has not been rigorously investigated in relation to schizotypy

- The current study did not find strong associations between MMN and schizotypy within a non-clinical population of young adults, but MMN was tended to be larger with higher suspiciousness scores

- This study raises questions regarding MMN as a marker of psychosis-risk in non-clinical populations 


\section{Introduction}

Schizophrenia is a psychiatric illness characterised by positive symptoms including hallucinations and delusions, negative symptoms such as avolition and alogia, and disorganised symptoms including bizarre behaviour and speech (APA, 2013). Whilst current antipsychotic treatments have moderate efficacy (Leucht et al., 2009), up to one third of patients remain symptomatic (van Os and Kapur, 2009). The aetiology of schizophrenia remains poorly understood, and the importance of moving from a categorical diagnosis to a dimensional approach is increasingly advocated by researchers within this field (David, 2010; Ettinger et al., 2014; Nelson et al., 2013).

One way in which this continuum might be understood is in terms of trait-like expressions of schizophrenia that are also present in varying degrees in the general population (Ettinger et al., 2014; Johns and van Os, 2001a). For example, schizotypy describes a cluster of personality traits which include unusual perceptual experiences, odd beliefs or magical thinking, odd or eccentric behaviour, social anxiety and isolation, constricted affect, suspiciousness and paranoia (Raine, 1991). Each of these is argued to map broadly onto the positive, negative and disorganised dimensions of schizophrenia (Fonseca-Pedrero et al., 2011; Nelson et al., 2013). Nelson et al. (2013) review competing models of schizotypy, including the quasidimensional approach which suggests that a small group of people, approximately $10 \%$ of the population known as "schizotypes", are vulnerable to schizophrenia (Lenzenweger, 2006; Meehl, 1990) and the fully dimensional approach (Claridge and Beech, 1995; Rawlings et al., 2008) in which schizotypy is conceptualised as existing along a continuum within the general population. The latter model is well supported in the literature (Johns and van Os, 2001b; Van Os et al., 2009), and is consistent with models describing continuity in clinical versus non-clinical symptoms of schizophrenia in the population (Linscott and Van Os, 2013; Linscott and Van Os, 2010). Neither model assumes that schizotypal traits are sufficient indicators of psychosis-risk, but rather that schizotypal traits interact with other environmental and genetic factors to confer risk (Nelson et al., 2013; Rawlings et al., 2008). Given the complexities of investigations into schizophrenia, including medication history, lifestyle factors such as substance use and smoking, and disease chronicity, research exploring schizotypy in the general population may provide information pertinent to the aetiology and risk factors associated with psychotic disorders (Nelson et al., 2013).

While there is a plethora of evidence from genetic studies in particular supporting the existence of schizotypy on a continuum with schizophrenia, increasing evidence also comes from neuropsychological and neurobiological research (for comprehensive reviews see,Ettinger et al., 2014; Nelson et al., 2013). For example, recent structural MRI work has indicated that high schizotypy is associated with reduced grey matter in the frontal and temporal lobes (DeRosse et al., 2014), reduced grey matter volume in medial prefrontal, orbitofrontal, and temporal cortical regions (Ettinger et al., 2012), and an aberrant neural prediction error signal (Corlett and Fletcher, 2012). Patients with schizotypal personality disorder showed altered auditory evoked potential N1, P2, N2 and P3 components intermediate between schizophrenia patients and healthy controls (Trestman et al., 1996). Schizotypy traits in a non-clinical population have also been associated with reduced P3 amplitude (Kimble et al., 2000), altered power within the electroencephalography (EEG) gamma frequency band (Kornmayer et al., 2015), sleep spindle density (Lustenberger et al., 2014), altered neural oscillations during a working memory task (Koychev et al., 2011), and impaired sensory gating as indicated by 
reduced P50 suppression (Croft et al., 2004; Croft et al., 2001; Evans et al., 2007). However, others have found a less clear pattern of findings; for example, Lagioia et al. (2010) did not find an association between default-mode activity and schizotypy in an adolescent sample. Modinos et al. (2010) also reported larger regional volumes in the medial posterior cingulate cortex (PCC) and the precuneus in individuals with high psychosis proneness as measured by the Community Assessment of Psychic Experiences (CAPE), which is contrary to findings in patients.

Intriguingly, despite a large body of research investigating alterations to the mismatch negativity (MMN) in patients with schizophrenia, a brain marker sensitive to N-methyl-D-aspartate (NMDA) receptor function (for a review see, Todd et al., 2013), very few studies have investigated the association between MMN alterations and schizotypy. MMN is a frontocentrally maximal event-related potential (ERP) component, defined as the point of greatest deviance following infrequent auditory (or visual) stimuli termed deviants, presented within a predictable pattern of frequent tones (or stimuli) (termed standard stimuli) (Kujala et al., 2007; Näätänen, 1995). The 'deviant' may differ from the 'standard' on a number of possible perceptual features including stimulus duration, frequency (pitch), intensity and location. The MMN is a component of the ERP visible within a difference waveform, computed by subtracting the brain response to the standard stimulus from the brain response elicited by the deviant (Picton et al., 2000), and is argued to reflect the evaluation of new auditory sensory input against current models of the acoustic environment (Garrido et al., 2009; Winkler, 2007). The two known generators of MMN, the superior temporal gyrus (STG) and inferior frontal gyrus (IFG; see Michie et al., 2016 for a review) are represented as a sum by mastoid-referenced MMN data recorded at frontal sites and may only be accurately dissociated with source localisation or computational approaches such as direct causal modelling (Garrido et al., 2009). Research in patients with schizophrenia, have consistently focused on MMN recorded at the frontocentral site electrode site ' $F z$ ', noting robust reductions (Umbricht and Krljes, 2005).

Robust reductions in MMN amplitude have been noted in patients with schizophrenia (Erickson et al., 2015; Näätänen and Kahkonen, 2009; Umbricht and Krljes, 2005), as well as their first degree relatives (Jessen et al., 2001; Michie et al., 2002; Sevik et al., 2011). These findings highlight the potential utility of MMN as a candidate endophenotype for the disorder (for a review of MMN as a marker of conferring vulnerability to schizophrenia, see Todd et al., 2013), although the evidence is mixed, (see, Bramon et al., 2004; Magno et al., 2008) and alterations to MMN have been noted in other disorders (for a review see, Näätänen et al., 2011). In patients, reductions in MMN have been linked with greater impairments in social and cognitive function (Näätänen et al., 2011), however evidence for an association between MMN and positive or negative symptoms of schizophrenia remains unclear (Todd et al., 2013; Umbricht and Krljes, 2005). MMN elicited by duration deviants (and to a lesser extent intensity deviants) has been linked in particular with the prodromal phase of illness (Todd et al., 2008), and is reduced in individuals at ultra-high risk of developing the disorder and patients with first episode psychosis (Atkinson et al., 2012; Bodatsch et al., 2011; Hermens et al., 2010; Hsieh et al., 2012; Kaur et al., 2011; Nagai et al.; Perez et al., 2014; Shaikh et al., 2012; Solis-Vivanco et al., 2014). Patients with chronic schizophrenia exhibit significant reductions in frequency MMN, with group differences for duration deviants less apparent at the later stages of illness (Todd et al., 2008). Grey matter loss in left and right Heschl's gyrus has been linked with attenuated frequency (Rasser et al., 2011; Salisbury et al., 2007) and duration MMN (Rasser et al., 2011) respectively in patients 
with schizophrenia. There is mounting evidence therefore that MMN may be sensitive to stage of illness in schizophrenia (i.e. attenuated duration MMN with the early stages of illness and reductions in frequency MMN with the later stages of illness) and indeed, that (duration) MMN deficits may identify at risk individuals who go on to develop psychosis (Atkinson et al., 2012; Bodatsch et al., 2011; Higuchi et al., 2013; Jahshan et al., 2012; Murphy et al., 2013; Perez et al., 2014). Therefore, $M M N$ is argued to be of particular interest with regard to future work targeting biomarkers of psychosis risk in vulnerable populations (Belger et al., 2012; Bodatsch et al., 2013; Light and Naatanen, 2013; Todd et al., 2013).

This work is already underway in individuals with schizotypal personality disorder features (Hong et al., 2012; Liu et al., 2007; Niznikiewicz et al., 2009), children at risk of developing schizophrenia (Bruggemann et al., 2013; Schreiber et al., 1992) and chronic cannabis users (e.g., Greenwood et al., 2014). Reduced MMN in a normative sample with high positive (schizotypal) symptom scores on the Personality Syndrome Questionnaire was reported in an abstract (Baldeweg et al., 1999), although the type of MMN was not reported. Fernandes and colleagues (1999) found no effect on frequency MMN amplitude of schizotypal symptoms, family history of psychosis, or group classification based on Chapman scales, in a normative sample. Although they did not measure MMN, Bates (2005) reported an association between auditory imprecision and higher schizotypal scores (measured using the Schizotypal Personality Questionnaire; SPQ) in a normative sample, reflecting reduced fidelity of primary sensory representations measured using a choice-reaction time task. Surprisingly, however, MMN has not been specifically investigated with respect to schizotypal personality traits in the general population as measured by the widely used SPQ, which has robust psychometric properties (Raine, 1991).

Therefore, the aim of the current study was to explore whether higher scores on the SPQ in a nonclinical sample were associated with attenuated MMN amplitude, consistent with the status of MMN as a biomarker for psychosis-proneness.

\section{Methods}

\subsection{Participants}

Participants were thirty-five undergraduate students (9 male, median 20.92 years of age, range: 18.8 to 37.6 years) from the University of Wollongong who were recruited via the School of Psychology's research participation scheme, as well as via flyers placed on noticeboards on the University campus and colleges. Exclusion criteria included any recent or regular (> twice weekly for a period of 6 months) illicit substance use, self-reported hearing impairments and neurological illness. In addition, all participants completed audiometric testing; all participants had normal hearing at $\leq 25 \mathrm{~dB}$ for $1000 \mathrm{~Hz}$ or $1500 \mathrm{~Hz}$ tones. All participants provided informed consent, and the study was approved by the Human Research Ethics Committee of the University of Wollongong. Participants received either course credit or AUD $\$ 30$ for their involvement in the study.

\subsection{Experimental paradigm and procedure}

Participants were familiarised with the study procedures before informed consent was obtained. A short structured interview was administered to participants to assess hearing or visual impairments, recent or regular substance use, and presence of any neurological or psychological disorder. Participants completed 
the full version of the Schizotypal Personality Questionnaire (SPQ, Raine, 1991), a 74 item measure of schizotypy, taking participants approximately 10 minutes to complete. SPQ scores on the nine subscales were calculated: Ideas of reference, Odd beliefs or magical thinking, Unusual perceptual experiences, Suspiciousness, Social anxiety, No close friends, Constricted affect, Odd behaviour, and Odd speech. In addition, dimensional SPQ scores were calculated in accordance with Raine et al. (1994). Specifically, the Cognitive-Perceptual dimension, which maps closely onto the positive symptoms of schizophrenia, was calculated based on the subscale scores for Ideas of reference, Odd beliefs or magical thinking, Unusual perceptual experiences, and Suspiciousness. The Interpersonal dimension, which has been linked with negative symptoms in schizophrenia, was comprised of the Social anxiety, No close friends and Constricted affect subscales, and the Disorganised dimension, linking with a similar set of symptoms in patients, was calculated using the Odd behaviour and Odd speech subscales.

In addition to the SPQ, participants completed the Beck Depression Inventory (BDI, Beck et al., 1961), State Trait Anxiety Inventory I and II (STAI, Spielberger et al., 1983), the Community Assessment of Psychic Experiences (CAPE, Stefanis et al., 2002) and the Oldfield Handedness Questionnaire (Oldfield, 1971) to assess symptoms of depression, anxiety, psychotic-like experiences and handedness, respectively (see Table 1). All participants also completed the Rey Auditory Verbal Learning Test (RAVLT), a measure of verbal learning and memory, before being fitted with recording electrodes. Participants completed an eye movement calibration task (Croft and Barry, 2000), and were instructed to sit as still as possible and to minimise blinking whilst completing a multifeature mismatch negativity paradigm (Greenwood et al., 2014) prior to two other EEG tasks not reported here. For further information on the multifeature paradigm, please see Supplement 1 and Greenwood et al. (2014).

\subsection{Mismatch negativity quantification}

For information on EEG data acquisition and processing, please see detailed methods in Supplement 1. MMN peak amplitude for each deviant condition was computed as the most negative peak within a 130-230 ms latency range for the duration condition, and 100-200 ms latency range for the frequency and intensity conditions. Quantification of the MMN was restricted to Fz only, as this component is largest at this site (Duncan et al., 2009), and attenuated MMN in patients with schizophrenia is most clearly observed at frontal scalp sites (Umbricht and Krljes, 2005).

\subsection{Data analysis}

As the SPQ results were not normally distributed, Spearman's correlations were used to explore the association between MMN and SPQ scores (including total, subscale and dimensional measures). In light of a trend level association between the Suspiciousness subscale and frequency MMN (see Figure 2 and section 3.2), a subsequent exploratory analysis was conducted comparing participants with high versus low Suspiciousness scores, divided using a median split.

Table 1 reports demographic, symptom and RAVLT performance measures for the High and Low Suspiciousness groups. Mann Whitney-U tests were used to compare the groups on demographic (age, gender), psychological (SPQ total and subscale scores, CAPE total frequency and distress, and subscale scores, BDI, STAI-I and II scores) and neuropsychological (RAVLT total score from trials 1 to 5) measures. 
MMN amplitude and latency data were entered into separate 2 (Group: High and Low Suspiciousness) by 3 (Condition: Duration, Frequency and Intensity) repeated measures ANOVA. Bonferroni corrected post-hoc tests were used to follow-up significant main effects and interactions.

\section{Results}

\subsection{Schizotypy scores in a non-clinical sample of young adults}

The median total SPQ score was 19 with a range of 0 to 56 (mean: 21.1, SD=14.95); 5 individuals received a score of 41 or more, indicating they fell within the previously suggested cut-off for severe proneness to schizotypal personality disorder (Raine, 1991). Table 1 reports the median and range for SPQ subscale and dimension scores.

\subsection{Is schizotypy associated with MMN in a non-clinical sample of young adults?}

Mismatch negativity difference waveforms at $\mathrm{Fz}$ for duration, frequency and intensity conditions are shown in Figure 1a and mastoid referenced grand mean ERP waveforms at $\mathrm{Fz}$ to the standard and deviant stimuli in Figure 1b. Spearman's correlations revealed no association between MMN amplitude in any condition and either the SPQ total score (all $p>0.52$ ), or the majority of subscale scores: Ideas of reference (all $p>0.52$ ), Social anxiety (all $p>0.33$ ), Odd beliefs or magical thinking (all $p>0.23$ ), Unusual perceptual experiences (all $p>0.21$ ), Odd behaviour (all $p>0.66$ ), Odd speech (all $p>0.35$ ), Constricted affect (all $p>0.25$ ) and No close friends (all $p>0.13$ ). There was however, a trend-level association between the Suspiciousness subscale and frequency MMN amplitude $\left(r_{s}(35)=-.306, p=0.073\right)$, with a non-significant association for duration $\left(r_{s}(35)=-.208, p=0.23\right)$ and intensity $\operatorname{MMN}\left(r_{s}(35)=-.158, p=0.37\right.$ see Figure 2$)$. No significant associations between SPQ dimensions and MMN amplitude in any condition were identified (Cognitive and Perceptual dimension: all $p>0.14$; Interpersonal dimension: all $p>0.62$; Disorganised dimension: all $p>0.48$ ).

In light of the trend-level association between Suspiciousness and MMN, we subsequently categorised individuals as high or low on Suspiciousness using a median split on Suspiciousness subscale scores, and conducted an exploratory between-groups analysis. The High and Low Suspiciousness groups ( $n=18$ and 17, respectively) did not differ significantly in age $(Z=-0.50, p=0.64)$, gender $\left(X^{2}=-0.83, p=0.77\right)$, state anxiety ( $Z=-0.98, p=0.35$ ), frequency or quantity of alcohol (frequency: $Z=-0.64, p=0.54$; quantity: $Z=-$ $0.78, p=0.46$ ) or tobacco use per month (frequency: $Z=-1.29, p=0.23$; quantity: $Z=-1.57, p=0.15$ ), nor performance on the RAVLT $(Z=-0.43, p=0.68)$. In addition to higher Suspiciousness subscale scores $(Z=-$ $5.18, p<0.001)$, the High Suspiciousness group reported significantly higher levels of depression $(Z=-2.36$, $p=0.02)$ and trait anxiety $(Z=-2.20, p=0.03)$, higher SPQ total $(Z=-3.57, p<0.001)$ and most SPQ subscale scores (Ideas of reference $(Z=-1.84, p=0.007)$; Social anxiety $(Z=-2.36, p=0.02)$; Unusual perceptual experiences $(Z=-3.15, p=0.002)$; Odd behaviour $(Z=-2.52, p=0.14)$; No close friends $(Z=-2.07, p=0.043)$; with a trend level difference for Constricted affect $(Z=-1.97, p=0.057)$, but no group difference for Odd speech $(Z=-$ 1.27, $p=0.22)$ ). The High Suspiciousness group also exhibited higher SPQ dimension scores (CognitivePerceptual dimension, $Z=-4.19, p<0.001$; Interpersonal dimension, $Z=-3.65, p<0.001$; trend level difference for Disorganised dimension; $Z=-1.79, p=0.077)$. Consistent with higher SPQ scores, CAPE total frequency $(Z=-$ $3.45, p=0.004)$ and distress scores $(Z=-2.36, p=0.02)$ were increased in the High Suspiciousness group, including higher frequency of positive symptoms $(Z=-2.55, p=0.01)$. In addition, the High Suspiciousness 
group reported higher frequency of negative and depressive symptoms and higher levels of distress associated with positive and depressive symptoms on the CAPE, however these group differences only reached trend level (all $p<0.10)$.

MMN difference waveforms at Fz for duration, frequency and intensity conditions for the High and Low Suspiciousness groups are shown in Figure 1c and mastoid referenced grand mean ERP waveforms at Fz to the standard and deviant stimuli in Figure 1d. Mean amplitude for standard ERPs over the latency windows used for MMN peak detection did not differ significantly between the groups.

Although for MMN amplitude, Condition was not found to interact significantly with Group ( $F<1)$, a main effect of Condition was observed $(F(2,66)=12.40, p<0.001)$ indicating $M M N$ amplitude was reduced in the frequency compared to the duration (Freq MMN M: $-2.90 \mu \mathrm{V}$, SD: $1.35 \mu \mathrm{V}$; Dur MMN M: $-4.53 \mu \mathrm{V}$, SD: $2.21 \mu \mathrm{V} ; \mathrm{p}<0.001$ ) and intensity conditions (Freq MMN M: -2.90 $\mu \mathrm{V}$, SD: $1.35 \mu \mathrm{V}$; Int MMN M: -4.07 $\mu \mathrm{V}$, SD: $2.18 \mu \mathrm{V} ; p<0.01)$. A significant effect of Group revealed larger $M M N$ amplitude, irrespective of deviant condition, in the High compared to the Low Suspiciousness group (High M: -4.35 $\mu \mathrm{V}$, SD: $2.02 \mu \mathrm{V}$; Low M: $3.29 \mu \mathrm{V}$, SD: $1.63 \mu \mathrm{V} ; F(1,33)=4.22, p=0.048)$.

There were no associations between MMN amplitude in any deviant condition and RAVLT performance (all $p>0.30$ ) or CAPE scores (all $p>0.09$ ).

\section{Discussion}

The current paper represents the first study to explore a potential association between auditory MMN and schizotypy in a non-clinical sample, in accord with a fully dimensional model of schizotypy and schizophrenia (Linscott and Van Os, 2013; Linscott and Van Os, 2010). This study was motivated by the increasing support in the literature for research into non-clinical schizotypy as a means of investigating the aetiology of schizophrenia whilst circumventing many of the complexities associated with conducting research in this population. Nevertheless, despite the robust reductions in MMN amplitude observed in patients with schizophrenia (Umbricht and Krljes, 2005), the current study did not find strong evidence for an association between schizotypy and the MMN. We did however, find a trend-level association between suspiciousness and MMN, such that, contrary to expectation, higher levels of suspiciousness were associated with increasingly larger MMN amplitude, and MMN was significantly larger (irrespective of deviant stimulus type) in a High compared to Low Suspiciousness group.

A consistent relationship between reduced $\mathrm{MMN}$ amplitude in schizophrenia and positive, negative or disorganised symptoms, has not been reported (for a discussion of this issue see (Todd et al., 2013) and meta-analysis (Umbricht and Krljes, 2005)). Instead, smaller MMN has been associated with impairments in global functioning (Kim et al., 2014; Light and Braff, 2005a, b; Rasser et al., 2011); an association that may be mediated by changes in brain structure and function, including grey matter loss (Rasser et al., 2011). Given that the current study comprised university students, it is likely that social and global functioning were in a normal range and reasonably homogenous in this group. Although MMN has long been argued a candidate endophenotype for schizophrenia, Todd et al. (2013) have reviewed existing evidence for this claim and note that while small MMN is reliably reported in patients, evidence for a phenotypic association with schizophrenia 
(based on the definition of endophenotype by Gottsman and Gould (2003)) and its independence from state and disease-process requires substantiative further study, best addressed by longitudinal work. Further, despite evidence that duration MMN is smaller in at risk groups (for example, those meeting at risk mental state (ARMS) criteria, Atkinson et al., 2012; Higuchi et al., 2013; Jahshan et al., 2012; Nagai et al.; Shaikh et al., 2012) and may predict transition to psychosis (Bodatsch et al., 2011; Higuchi et al., 2013; Perez et al., 2014; Shaikh et al., 2012), Todd et al. (2013) highlight that it is currently unknown whether the reductions in MMN observed in the ARMS group reflect psychosis-risk or alternatively, may reflect the early stages of disease progression. If schizotypy reflects one end of a continuum with schizophrenia as described by a fully dimensional model, it follows that smaller MMN should be associated with increasing schizotypy traits in the general population. The current data however, do not support this hypothesis. An alternative hypothesis is that attenuated MMN amplitude may be more specifically related to the disease state of schizophrenia and not to schizotypy scores (or this belief / personality trait structure per se) in non-clinical populations.

Although there is not a large body of electrophysiological studies pertinent to schizotypy, interestingly, we are not the first study to report findings opposite to those observed in patients with schizophrenia. Importantly, larger frontal MMN to frequency deviants was reported in patients with schizotypal personality disorder, though not paranoid personality disorder (Liu et al., 2007). In contrast to Bates (2005) showing an association between higher schizotypal scores and auditory imprecision in a normative sample, Liu et al. (2007) showed that schizotypal patients had normal discrimination of auditory stimuli and suggested that their findings might reflect difficulty in filtering irrelevant stimuli. The MMN has been argued to reflect the brain's capacity to 'gate in' novel or new information (Boutros and Belger, 1999; Gjini et al., 2010), so it seems more likely that larger MMN amplitudes might reflect compensatory processes possibly related to difficulty filtering relevant from irrelevant auditory stimuli. In a study of treatment-refractory schizophrenia, in conjunction with atypical topography, larger frequency MMN amplitude was also observed in patients relative to controls (Milovan et al., 2004), which in light of the component's unusual topography may indeed reflect compensatory processing. More recently, a visual component similar to the auditory MMN was also enhanced in high compared to low scorers on the O-LIFE questionnaire (Mason and Claridge, 2006) which the authors also argue might reflect compensatory processes due to deficient executive functioning (Fuggetta et al., 2015). Whilst these data are inconsistent with otherwise widely reported reductions in MMN in patients with schizophrenia (Erickson et al., 2015; Umbricht and Krljes, 2005), they highlight the complexity and heterogeneity within 'at risk' samples, including schizotypy.

A further hypothesis is that larger MMN amplitudes in the high Suspiciousness group may reflect heightened involuntary attention, possibility related to distractibility (Näätänen et al., 2012), aberrant salience (eg., Kornmayer et al., 2015) and altered early perceptual processing (Sumich et al., 2014). There is some evidence that higher levels of suspiciousness may be linked with aberrant perceptual processing in nonclinical populations, and this may lead to ambiguous stimuli being interpreted as threatening (Sumich et al., 2014) and may be related to changes in grey matter volume in left Heschl gyrus, a possible generator of MMN (Kasai et al., 2003). Suspiciousness has been associated with heightened neural response, N200, to emotional stimuli, regardless of specific emotion content (Fisher et al., 2014). Further, previous research in patients with (clinically diagnosed) schizotypal personality disorder exhibited altered auditory evoked potentials, with N1, P2, N2 and P3 components at levels which were intermediate to patients with 
schizophrenia and healthy controls (Trestman et al., 1996). Whereas research examining the underlying brain structures may facilitate interpretation of these electrophysiological findings, some neuroimaging studies have noted further disparate findings. For example, structural neuroimaging studies have shown, in contrast to schizophrenia patient data, that (non-clinical) subjects with high positive schizotypy had larger grey matter regional volumes in the medial posterior cingulate cortex (PCC) and the precuneus (Modinos et al. (2010). One possible interpretation put forward by the authors was that increased grey matter volume in young adults with high schizotypy might reflect delayed synaptic pruning (Modinos et al., 2010), consistent with the idea that schizophrenia may be associated with altered neural development during adolescence (Rapoport et al., 2005). Previously, grey matter reductions in frontotemporal regions have been linked with MMN reduction in patients with schizophrenia (Rasser et al., 2011). In light of the young median age of our sample (21 years) and the proposed relationship between MMN and NMDA receptor function, it is possible that our findings may reflect delayed brain maturational processes, similarly to what was reported by Modinos et al. (2010). Nevertheless, the current data continue to raise questions regarding the extent to which neurobiological alterations associated with schizophrenia are similarly observed in schizotypy.

There are a number of limitations to the current study: First the results of the current study are limited by the small sample size, exploratory nature of our analyses, and overrepresentation of female compared to male participants. As very little research has examined the association between MMN and schizotypy in nonclinical adults, there is significant scope for future research to build on this study. It will be important to replicate the non-significant findings in a large community sample, as it is possible that unmeasured 'resilience' factors, such as social and global functioning, are over-represented in the current sample of undergraduate students. Second, in this study we focused solely on the MMN as a marker of risk. These results could be strengthened in future studies if other candidate neurobiological markers of psychosisproneness such as the P50 (Bramon et al., 2004; de Wilde et al., 2007) and P300 (Bramon et al., 2005) were also collected. The latter, especially an analysis of $\mathrm{P} 3 \mathrm{a}$, might inform a possible increased involuntary attention response in a schizotypy sample. Additionally, in the current study we re-referenced our ERP data to the mastoids and focused our analysis on the frontocentral MMN component. Future research would benefit from additional and separate analysis of the temporal generators of the $\mathrm{MMN}$ for which source localisation procedures would be required. Third, consistent with the argument that biological markers of psychosis risk likely reflect underlying genetic factors common across schizotypy and schizophrenia, while it is the interaction of these with environmental factors that causes individuals to go on to develop schizophrenia, including data about potential environmental factors and candidate genes with measures of MMN could help to elucidate the relationship between MMN, schizotypy traits, and the pathophysiology of schizophrenia (eg., Baker et al., 2005). Finally, it is possible that the associations with Suspiciousness observed in the current study and psychological symptom differences between the High and Low Suspiciousness group may not be specific to Suspiciousness per se and instead reflect a more general psychosis-prone phenotype. This limitation of the current study is worthy of further investigation with a larger number of measures, including broader measures of psychosis-proneness eg., the O-LIFE scale (Mason and Claridge, 2006).

In summary, the current paper reflects an exploratory investigation of an association between MMN and schizotypy in a sample of non-clinical young adults. We found a larger MMN in individuals with High compared to Low Suspiciousness, a pattern which is in direct contrast to attenuated MMN observed in 
patients. This finding is consistent with larger frequency MMN reported in clinical sample of patients with schizotypal personality disorder (Liu et al. 2007). Importantly, in our study larger MMN amplitudes were found within a non-clinical sample of young adults with higher Suspiciousness scores, and irrespective of MMN deviant type. Given the robust literature of decreased $M M N$ in patients with schizophrenia these data warrant replication, as they raise questions regarding the (i) validity of the MMN as a marker of susceptibility in nonclinical and low risk populations and (ii) the relationship between schizotypy scores in non-clinical populations and risk for schizophrenia. 


\section{Acknowledgement.}

The authors would like to thank Kaitlin Saunders, Felicity Webster and Camilla Beale for their assistance in data-collection and participant recruitment. 


\section{References}

APA, 2013. Diagnostic and statistical manual of mental disorders. 5th Edition American Psychiatric Association, Washington, DC.

Atkinson, R.J., Michie, P.T., Schall, U., 2012. Duration mismatch negativity and P3a in first-episode psychosis and individuals at ultra-high risk of psychosis. Biol. Psychiatry 71, 98-104.

Baker, K., Baldeweg, T., Sivagnanasundaram, S., Scambler, P., Skuse, D., 2005. COMT Val108/158 Met modifies mismatch negativity and cognitive function in 22q11 deletion syndrome. Biol. Psychiatry 58 , 23-31.

Baldeweg, T., Adams, S., Gruzelier, J., 1999. Mismatch negativity (MMN) in relation to positive and negative features of schizotypy and extremes of pubertal timing. Schizophr. Res. 36, 251-251.

Bates, T.C., 2005. The panmodal sensory imprecision hypothesis of schizophrenia: reduced auditory precision in schizotypy. Pers. Indiv. Differ. 38, 437-449.

Beck, A.T., Ward, C.H., Mendelson, M., Mock, J., Erbaugh, J., 1961. An inventory for measuring depression. Arch. Gen. Psychiatry 4, 561-571.

Belger, A., Yucel, G.H., Donkers, F.C.L., 2012. In search of psychosis biomarkers in high-risk populations: Is the mismatch negativity the one we've been waiting for? Biol. Psychiatry 71, 94-95.

Bodatsch, M., Klosterkotter, J., Daumann, J., 2013. Contributions of experimental psychiatry to research on the psychosis prodrome. Front. Psychiatry 4, 170.

Bodatsch, M., Ruhrmann, S., Wagner, M., Muller, R., Schultze-Lutter, F., Frommann, I., Brinkmeyer, J., Gaebel, W., Maier, W., Klosterkotter, J., Brockhaus-Dumke, A., 2011. Prediction of psychosis by mismatch negativity. Biol. Psychiatry 69, 959-966.

Boutros, N.N., Belger, A., 1999. Midlatency evoked potentials attenuation and augmentation reflect different aspects of sensory gating. Biol. Psychiatry 45, 917-922.

Bramon, E., Croft, R.J., McDonald, C., Virdi, G.K., Gruzelier, J.G., Baldeweg, T., Sham, P.C., Frangou, S., Murray, R.M., 2004. Mismatch negativity in schizophrenia: a family study. Schizophr. Res. 67, 1-10.

Bramon, E., McDonald, C., Croft, R.J., Landau, S., Filbey, F., Gruzelier, J.H., Sham, P.C., Frangou, S., Murray, R.M., 2005. Is the P300 wave an endophenotype for schizophrenia? A meta-analysis and a family study. Neuroimage 27, 960-968.

Bruggemann, J.M., Stockill, H.V., Lenroot, R.K., Laurens, K.R., 2013. Mismatch negativity (MMN) and sensory auditory processing in children aged 9-12 years presenting with putative antecedents of schizophrenia. Int. J. Psychophysiol. 89, 374-380.

Claridge, G., Beech, T., 1995. constructions of schizotypy. Schizotypal personality, 192.

Corlett, P.R., Fletcher, P.C., 2012. The neurobiology of schizotypy: fronto-striatal prediction error signal correlates with delusion-like beliefs in healthy people. Neuropsychologia 50, 3612-3620.

Croft, R.J., Barry, R.J., 2000. EOG correction: Which regression should we use? Psychophysiology 37, 123125.

Croft, R.J., Dimoska, A., Gonsalvez, C.J., Clarke, A.R., 2004. Suppression of P50 evoked potential component, schizotypal beliefs and smoking. Psychiatry Res. 128, 53-62. 
Croft, R.J., Lee, A., Bertolot, J., Gruzelier, J.H., 2001. Associations of P50 suppression and desensitization with perceptual and cognitive features of "unreality" in schizotypy. Biol. Psychiatry 50, 441-446.

David, A.S., 2010. Why we need more debate on whether psychotic symptoms lie on a continuum with normality. Psychol. Med. 40, 1935-1942.

de Wilde, O.M., Bour, L.J., Dingemans, P.M., Koelman, J.H., Linszen, D.H., 2007. A meta-analysis of P50 studies in patients with schizophrenia and relatives: differences in methodology between research groups. Schizophr. Res. 97, 137-151.

DeRosse, P., Nitzburg, G.C., Ikuta, T., Peters, B.D., Malhotra, A.K., Szeszko, P.R., 2014. Evidence from structural and diffusion tensor imaging for frontotemporal deficits in psychometric schizotypy. Schizophr Bull.

Duncan, C.C., Barry, R.J., Connolly, J.F., Fischer, C., Michie, P.T., Näätänen, R., Polich, J., Reinvang, I., Peeten, C.V., 2009. Event-related potentials in clinical research: Guidelines for eliciting, recording and quantifying mismatch negativity, P300, and N400. Clin. Neuropsychiatry 120, 1883-1908.

Erickson, M.A., Ruffle, A., Gold, J.M., 2015. A meta-analysis of mismatch negativity in schizophrenia: From clinical risk to disease specificity and progression. Biol. Psychiatry.

Ettinger, U., Meyhofer, I., Steffens, M., Wagner, M., Koutsouleris, N., 2014. Genetics, cognition, and neurobiology of schizotypal personality: a review of the overlap with schizophrenia. Front. Psychiatry $5,18$.

Ettinger, U., Williams, S.C., Meisenzahl, E.M., Moller, H.J., Kumari, V., Koutsouleris, N., 2012. Association between brain structure and psychometric schizotypy in healthy individuals. World J. Biol. Psychiatry 13, 544-549.

Evans, L.H., Gray, N.S., Snowden, R.J., 2007. Reduced P50 suppression is associated with the cognitive disorganisation dimension of schizotypy. Schizophr. Res. 97, 152-162.

Fernandes, L.O., Keller, J., Giese-Davis, J.E., Hicks, B.D., Klein, D.N., Miller, G.A., 1999. Converging evidence for a cognitive anomaly in early psychopathology. Psychophysiology 36, 511-521.

Fonseca-Pedrero, E., Paino, M., Lemos-Giraldez, S., Sierra-Baigrie, S., Muniz, J., 2011. Measurement invariance of the Schizotypal Personality Questionnaire-Brief across gender and age. Psychiatry Res. 190, 309-315.

Fuggetta, G., Bennett, M.A., Duke, P.A., 2015. An electrophysiological insight into visual attention mechanisms underlying schizotypy. Biol. Psychol. 109, 206-221.

Garrido, M.I., Kilner, J.M., Stephan, K.E., Friston, K.J., 2009. The mismatch negativity: A review of underlying mechanisms. Clin. Neurophysiology 120, 453-463.

Gjini, K., Arfken, C., Boutros, N.N., 2010. Relationships between sensory "gating out" and sensory "gating in" of auditory evoked potentials in schizophrenia: a pilot study. Schizophr. Res. 121, 139-145.

Gottesman, I.I., Gould, T.D., 2003. The endophenotype concept in psychiatry: etymology and strategic intentions. Am. J. Psychiatry 160, 636-645.

Greenwood, L.M., Broyd, S.J., Croft, R., Todd, J., Michie, P.T., Johnstone, S., Murray, R., Solowij, N., 2014. Chronic Effects of Cannabis Use on the Auditory Mismatch Negativity. Biol. Psychiatry 75, 449-458. 
Hermens, D.F., Ward, P.B., Hodge, M.A., Kaur, M., Naismith, S.L., Hickie, I.B., 2010. Impaired MMN/P3a complex in first-episode psychosis: cognitive and psychosocial associations. Prog. Neuropsychopharm. Biol. Psychiatry 34, 822-829.

Higuchi, Y., Sumiyoshi, T., Seo, T., Miyanishi, T., Kawasaki, Y., Suzuki, M., 2013. Mismatch negativity and cognitive performance for the prediction of psychosis in subjects with at-risk mental state. PLoS One 8, e54080.

Hong, L.E., Moran, L.V., Du, X., O'Donnell, P., Summerfelt, A., 2012. Mismatch negativity and low frequency oscillations in schizophrenia families. Clin Neurophysiol 123, 1980-1988.

Hsieh, M.H., Shan, J.C., Huang, W.L., Cheng, W.C., Chiu, M.J., Jaw, F.S., Hwu, H.G., Liu, C.C., 2012. Auditory event-related potential of subjects with suspected pre-psychotic state and first-episode psychosis. Schizophr. Res. 140, 243-249.

Jahshan, C., Cadenhead, K.S., Rissling, A.J., Kirihara, K., Braff, D.L., Light, G.A., 2012. Automatic sensory information processing abnormalities across the illness course of schizophrenia. Psychol. Med. 42, 85-97.

Jessen, F., Fries, T., Kucharski, C., Nishimura, T., Hoenig, K., Maier, W., Falkai, P., Heun, R., 2001. Amplitude reduction of the mismatch negativity in first-degree relatives of patients with schizophrenia. Neurosci. Lett. 309, 185-188.

Johns, L.C., van Os, J., 2001. The continuity of psychotic experiences in the general population. Clin. Psychol. Rev. 21, 1125-1141.

Kaur, M., Battisti, R.A., Ward, P.B., Ahmed, A., Hickie, I.B., Hermens, D.F., 2011. MMN/P3a deficits in first episode psychosis: comparing schizophrenia-spectrum and affective-spectrum subgroups. Schizophr. Res. 130, 203-209.

Kim, M., Kim, S.N., Lee, S., Byun, M.S., Shin, K.S., Park, H.Y., Jang, J.H., Kwon, J.S., 2014. Impaired mismatch negativity is associated with current functional status rather than genetic vulnerability to schizophrenia. Psychiatry Res. Neuroimaging 222, 100-106.

Kimble, M., Lyons, M., O'Donnell, B., Nestor, P., Niznikiewicz, M., Toomey, R., 2000. The effect of family status and schizotypy on electrophysiologic measures of attention and semantic processing. Biol. Psychiatry 47, 402-412.

Kornmayer, L., Leicht, G., Mulert, C., 2015. Increased gamma oscillations evoked by physically salient distracters are associated with schizotypy. Brain Topogr. 28, 153-161.

Koychev, I., Deakin, J.F., Haenschel, C., El-Deredy, W., 2011. Abnormal neural oscillations in schizotypy during a visual working memory task: support for a deficient top-down network? Neuropsychologia 49, 2866-2873.

Kujala, T., Tervaniemi, M., Schröger, E., 2007. The mismatch negativity in cognitive and clinical neuroscience: Theoretical and methodological considerations. Biol. Psychol. 74, 1-19.

Lagioia, A., Van De Ville, D., Debbane, M., Lazeyras, F., Eliez, S., 2010. Adolescent resting state networks and their associations with schizotypal trait expression. Front. Systems Neurosci. 4.

Lenzenweger, M.F., 2006. Schizotaxia, schizotypy, and schizophrenia: Paul E. Meehl's blueprint for the experimental psychopathology and genetics of schizophrenia. J. Abnorm. Psychol. 115, 195-200. 
Leucht, S., Arbter, D., Engel, R.R., Kissling, W., Davis, J.M., 2009. How effective are second-generation antipsychotic drugs? A meta-analysis of placebo-controlled trials. Mol. Psychiatry 14, 429-447.

Light, G.A., Braff, D.L., 2005a. Mismatch negativity deficits are associated with poor functioning in schizophrenia patients. Arch. Gen. Psychiatry 62, 127-136.

Light, G.A., Braff, D.L., 2005b. Stability of mismatch negativity deficits and their relationship to functional impairments in chronic schizophrenia. Am. J. Psychiatry 162, 1741-1743.

Light, G.A., Naatanen, R., 2013. Mismatch negativity is a breakthrough biomarker for understanding and treating psychotic disorders. Proc. Natl. Acad. Sci. U.S.A. 110, 15175-15176.

Linscott, R., Van Os, J., 2013. An updated and conservative systematic review and meta-analysis of epidemiological evidence on psychotic experiences in children and adults: on the pathway from proneness to persistence to dimensional expression across mental disorders. Psychol. Med. 43, 1133-1149.

Linscott, R.J., Van Os, J., 2010. Systematic reviews of categorical versus continuum models in psychosis: evidence for discontinuous subpopulations underlying a psychometric continuum. Implications for DSM-V, DSM-VI, and DSM-VII. Annu. Rev. Clin. Psychol. 6, 391-419.

Liu, Y., Shen, X., Zhu, Y., Xu, Y., Cai, W., Shen, M., Yu, R., Wang, W., 2007. Mismatch negativity in paranoid, schizotypal, and antisocial personality disorders. Clin. Neurophysiology 37, 89-96.

Lustenberger, C., O'Gorman, R.L., Pugin, F., Tushaus, L., Wehrle, F., Achermann, P., Huber, R., 2014. Sleep spindles are related to schizotypal personality traits and thalamic glutamine/glutamate in healthy subjects. Schizophr. Bull.

Magno, E., Yeap, S., Thakore, J.H., Garavan, H., De Sanctis, P., Foxe, J.J., 2008. Are auditory-evoked frequency and duration mismatch negativity deficits endophenotypic for schizophrenia? High-density electrical mapping in clinically unaffected first-degree relatives and first-episode and chronic schizophrenia. Biol. Psychiatry 64, 385-391.

Mason, O., Claridge, G., 2006. The Oxford-Liverpool Inventory of Feelings and Experiences (O-LIFE): further description and extended norms. Schizophr. Res. 82, 203-211.

Meehl, P.E., 1990. Toward an integrated theory of schizotaxia, schizotypy, and schizophrenia. J. Pers. Disord. 4, 1-99.

Michie, P.T., Innes-Brown, H., Todd, J., Jablensky, A.V., 2002. Duration mismatch negativity in biological relatives of patients with schizophrenia spectrum disorders. Biol. Psychiatry 52, 749-758.

Michie, P.T., Malmierca, M.S., Harms, L., Todd, J., 2016. The neurobiology of MMN and implications for schizophrenia. Biol. Psychol. 116, 90-97.

Milovan, D.L., Baribeau, J., Roth, R.M., Stip, E., 2004. ERP study of pre-attentive auditory processing in treatment-refractory schizophrenia. Brain Cogn. 55, 355-357.

Modinos, G., Mechelli, A., Ormel, J., Groenewold, N.A., Aleman, A., McGuire, P.K., 2010. Schizotypy and brain structure: a voxel-based morphometry study. Psychol. Med. 40, 1423-1431.

Murphy, J.R., Rawdon, C., Kelleher, I., Twomey, D., Markey, P.S., Cannon, M., Roche, R.A.P., 2013. Reduced duration mismatch negativity in adolescents with psychotic symptoms: further evidence for mismatch negativity as a possible biomarker for vulnerability to psychosis. BMC. Psychiatry 13 . 
Näätänen, R., 1995. The mismatch negativity: A powerful tool for cognitive neuroscience. Ear and Hearing 16, 6-18.

Näätänen, R., Kahkonen, S., 2009. Central auditory dysfunction in schizophrenia as revealed by the mismatch negativity (MMN) and its magnetic equivalent MMNm: a review. Int. J. Neuropsychopharmacol. 12, 125-135.

Näätänen, R., Kujala, T., Escera, C., Baldeweg, T., Kreegipuu, K., Carlson, S., Ponton, C., 2012. The mismatch negativity (MMN) - A unique window to disturbed central auditory processing in ageing and different clinical conditions Clin. Neurophysiology 123, 424-458.

Näätänen, R., Kujala, T., Kreegipuu, K., Carlson, S., Escera, C., Baldeweg, T., Ponton, C., 2011. The mismatch negativity: an index of cognitive decline in neuropsychiatric and neurological diseases and in ageing. Brain 134, 3435-3453.

Nagai, T., Tada, M., Kirihara, K., Araki, T., Jinde, S., Kasai, K., Mismatch negativity as a "translatable" brain marker toward early intervention for psychosis: A review. Front. Psychiatry. 2013 Sep 23;4:115.

Nelson, M.T., Seal, M.L., Pantelis, C., Phillips, L.J., 2013. Evidence of a dimensional relationship between schizotypy and schizophrenia: a systematic review. Neurosci. Biobehav. Rev. 37, 317-327.

Niznikiewicz, M.A., Spencer, K.M., Dickey, C., Voglmaier, M., Seidman, L.J., Shenton, M.E., McCarley, R.W., 2009. Abnormal pitch mismatch negativity in individuals with schizotypal personality disorder. Schizophr. Res. 110, 188-193.

Oldfield, R.C., 1971. The assessment and analysis of handedness: The edinburgh inventory. Neuropsychololgia 9, 97-113.

Perez, V.B., Woods, S.W., Roach, B.J., Ford, J.M., McGlashan, T.H., Srihari, V.H., Mathalon, D.H., 2014. Automatic auditory processing deficits in schizophrenia and clinical high-risk patients: forecasting psychosis risk with mismatch negativity. Biol. Psychiatry 75, 459-469.

Picton, T.W., Alain, C., Otten, L., Ritter, W., Achim, A., 2000. Mismatch negativity: different water in the same river. Audiol. Neurotol. 5, 111-139.

Raine, A., 1991. The SPQ: A scale for the assessment of schizotypal personality based on DSM-III-R criteria. Schizophr. Bull. 17, 555-564.

Raine, A., Reynolds, C., Lencz, T., Scerbo, A., Triphon, N., Kim, D., 1994. Cognitive-Perceptual, Interpersonal, and Disorganized Features of Schizotypal Personality. Schizophr. Bull. 20, 191-201.

Rapoport, J.L., Addington, A.M., Frangou, S., Psych, M.R.C., 2005. The neurodevelopmental model of schizophrenia: update 2005. Mol. Psychiatry 10, 434-449.

Rasser, P.E., Schall, U., Todd, J., Michie, P.T., Ward, P.B., Johnston, P., Helmbold, K., Case, V., Soyland, A., Tooney, P.A., Thompson, P.M., 2011. Gray matter deficits, mismatch negativity, and outcomes in schizophrenia. Schizophr. Bull. 37, 131-140.

Rawlings, D., Williams, B., Haslam, N., Claridge, G., 2008. Is schizotypy taxonic? Response to Beauchaine, Lenzenweger, and Waller. Pers. Indiv. Differ. 44, 1663-1672.

Salisbury, D.F., Kuroki, N., Kasai, K., Shenton, M.E., McCarley, R.W., 2007. Progressive and interrelated functional and structural evidence of post-onset brain reduction in schizophrenia. Arch. Gen. Psychiatry 64, 521-529. 
Schreiber, H., Stolz-Born, G., Kornhuber, H.H., Born, J., 1992. Event-related potential correlates of impaired selective attention in children at high risk for schizophrenia. Biol. Psychiatry 32, 634-651.

Sevik, A.E., Yagcioglu, A.E.A., Yagcioglu, S., Karahan, S., Gurses, N., Yildiz, M., 2011. Neuropsychological performance and auditory event related potentials in schizophrenia patients and their siblings: A family study. Schizophr. Res. 130, 195-202.

Shaikh, M., Valmaggia, L., Broome, M.R., Dutt, A., Lappin, J., Day, F., Woolley, J., Tabraham, P., Walshe, M., Johns, L., Fusar-Poli, P., Howes, O., Murray, R.M., McGuire, P., Bramon, E., 2012. Reduced mismatch negativity predates the onset of psychosis. Schizophr. Res. 134, 42-48.

Solis-Vivanco, R., Mondragon-Maya, A., Leon-Ortiz, P., Rodriguez-Agudelo, Y., Cadenhead, K.S., de la Fuente-Sandoval, C., 2014. Mismatch Negativity reduction in the left cortical regions in first-episode psychosis and in individuals at ultra high-risk for psychosis. Schizophr. Res. 158, 58-63.

Spielberger, C.D., Gorsuch, R.L., Lushene, R., Vagg, P.R., Jacobs, G.A., 1983. Manual for the state-trait anxiety inventory. Consulting psychologists press, Palo Alto, CA.

Stefanis, N., Hanssen, M., Smirnis, N., Avramopoulos, D., Evdokimidis, I., Stefanis, C., Verdoux, H., Os, V.J., 2002. Evidence that three dimensions of psychosis have a distribution in the general population. Psychol. Med. 32, 347-358.

Sumich, A., Castro, A., Kumari, V., 2014. N100 and N200, but not P300, amplitudes predict paranoia/suspiciousness in the general population. Pers. Indiv. Differ. 61, 74-79.

Todd, J., Harms, L., Michie, P., Schall, U., 2013. Mismatch negativity (MMN): Translating the potential. Front. Psychiatry 4.

Todd, J., Michie, P.T., Schall, U., Karayanidis, F., Yabe, H., Naatanen, R., 2008. Deviant matters: Duration, frequency, and intensity deviants reveal different patterns of mismatch negativity reduction in early and late schizophrenia. Biol. Psychiatry 63, 58-64.

Trestman, R.L., Horvath, T., Kalus, O., Peterson, A.E., Coccaro, E., Mitropoulou, V., Apter, S., Davidson, M., Siever, L.J., 1996. Event-related potentials in schizotypal personality disorder. J. Neuropsychiatry Clin. Neurosci. 8, 33-40.

Umbricht, D., Krljes, S., 2005. Mismatch negativity in schizophrenia: a meta-analysis. Schizophr. Res. 76, 123.

van Os, J., Kapur, S., 2009. Schizophrenia. Lancet 374, 635-645.

Van Os, J., Linscott, R.J., Myin-Germeys, I., Delespaul, P., Krabbendam, L., 2009. A systematic review and meta-analysis of the psychosis continuum: evidence for a psychosis proneness-persistenceimpairment model of psychotic disorder. Psychol. Med. 39, 179-195.

Winkler, I., 2007. Interpreting the mismatch negativity. J. Psychophysiol. 21, 147-163. 
Table 1. Participant demographic information, cognitive and psychological symptom measures

\begin{tabular}{|c|c|c|c|c|c|c|c|c|c|}
\hline & \multicolumn{3}{|c|}{ Whole sample } & \multicolumn{3}{|c|}{ Low Suspiciousness } & \multicolumn{3}{|c|}{ High Suspiciousness } \\
\hline & $\mathbf{N}$ & Median & [Range] & $\mathbf{N}$ & Median & [Range] & $\mathbf{N}$ & Median & [Range] \\
\hline Age & 35 & 20.92 & [18.75-37.55] & 17 & 20.48 & [19.14-37.55] & 18 & 20.96 & {$[18.75-27.23]$} \\
\hline Gender & 35 & $9 \mathrm{M} / 26 \mathrm{~F}$ & & 17 & $4 \mathrm{M} / 13 \mathrm{~F}$ & & 18 & $5 \mathrm{M} / 13 \mathrm{~F}$ & \\
\hline Alcohol frequency ${ }^{a}$ & 30 & 2.50 & {$[0-30]$} & 17 & 2.50 & {$[0-30]$} & 13 & 2.50 & {$[0.4-30]$} \\
\hline Alcohol quantity ${ }^{b}$ & 30 & 9.50 & {$[0-165]$} & 17 & 8.13 & {$[0-42]$} & 13 & 10.00 & [1.4-165] \\
\hline Cigarette frequency ${ }^{a}$ & 17 & 0.20 & {$[0-30]$} & 10 & 0.00 & {$[0-30]$} & 7 & 1.00 & {$[0-30]$} \\
\hline Cigarette quantity ${ }^{b}$ & 14 & 1.00 & {$[0-450]$} & 9 & 0.00 & [0-450] & 5 & 1.00 & {$[1-270]$} \\
\hline RAVLT total words trials I to $\mathrm{V}$ & 35 & 59.00 & [38-68] & 17 & 59.00 & [38-67] & 18 & 59.00 & [48-68] \\
\hline BDI & 35 & 4.00 & {$[0-17]$} & 17 & 2.00 & {$[0-11]$} & 18 & 5.00 & {$[0-17]^{*}$} \\
\hline STAI state & 34 & 32.50 & [19-57] & 16 & 29.00 & [19-57] & 18 & 32.50 & [20-49] \\
\hline STAI trait & 35 & 39.00 & [21-62] & 17 & 35.00 & [21-53] & 18 & 45.50 & {$[24-62]^{*}$} \\
\hline SPQ Total & 35 & 19.00 & {$[0-56]$} & 17 & 12.00 & {$[0-21]$} & 18 & 30.20 & {$[3-56]^{\star *}$} \\
\hline SPQ Ideas of reference & 35 & 2.00 & {$[0-8]$} & 17 & 1.00 & {$[0-4]$} & 18 & 4.00 & {$[0-8]^{\star *}$} \\
\hline SPQ Excessive social anxiety & 35 & 3.00 & {$[0-8]$} & 17 & 2.00 & {$[0-7]$} & 18 & 5.00 & {$[0-8]^{*}$} \\
\hline SPQ Odd beliefs or magical thinking & 35 & 0.00 & {$[0-5]$} & 17 & 0.00 & {$[0-5]$} & 18 & 1.00 & {$[0-5]^{\dagger}$} \\
\hline SPQ Unusual perceptual experiences & 35 & 1.00 & {$[0-9]$} & 17 & 0.00 & {$[0-3]$} & 18 & 2.50 & {$[0-9]^{*}$} \\
\hline SPQ Odd or eccentric behaviour & 35 & 1.00 & {$[0-7]$} & 17 & 0.00 & {$[0-4]$} & 18 & 2.50 & {$[0-7]^{*}$} \\
\hline SPQ No close friends & 35 & 2.00 & {$[0-7]$} & 17 & 1.00 & {$[0-5]$} & 18 & 3.50 & {$[0-7]^{*}$} \\
\hline SPQ Odd speech & 35 & 4.00 & {$[0-9]$} & 17 & 3.00 & {$[0-6]$} & 18 & 4.00 & {$[0-9]$} \\
\hline SPQ Constricted affect & 35 & 1.00 & {$[0-7]$} & 17 & 1.00 & {$[0-4]$} & 18 & 1.50 & {$[0-7]^{*}$} \\
\hline SPQ Suspiciousness & 35 & 2.00 & {$[0-8]$} & 17 & 0.00 & {$[0-1]$} & 18 & 4.00 & {$[2-8]^{\star *}$} \\
\hline SPQ Cognitive-Perceptual dimension ${ }^{c}$ & 35 & 6.00 & {$[0-23]$} & 17 & 2.00 & {$[0-10]$} & 18 & 14.20 & {$[2-23]^{\star *}$} \\
\hline SPQ Interpersonal dimensions & 35 & 9.00 & {$[0-30]$} & 17 & 5.00 & {$[0-15]$} & 18 & 14.00 & {$[3-30]^{\star *}$} \\
\hline SPQ Disorganised dimension & 35 & 4.00 & {$[0-15]$} & 17 & 4.00 & {$[0-10]$} & 18 & 6.50 & {$[0-15]^{\dagger}$} \\
\hline CAPE Total frequency & 35 & 63.00 & [41-104] & 17 & 59.00 & [42-73] & 18 & 68.00 & {$[53-104]^{*}$} \\
\hline CAPE Total distress & 35 & 28.00 & {$[4-82]$} & 17 & 23.00 & {$[4-63]$} & 18 & 36.00 & {$[11-82]^{*}$} \\
\hline
\end{tabular}




\begin{tabular}{|c|c|c|c|c|c|c|c|c|c|}
\hline CAPE Negative frequency & 35 & 24.00 & [14-41] & 17 & 22.00 & [14-30] & 18 & 25.50 & {$[18-41]^{\dagger}$} \\
\hline CAPE Negative distress & 35 & 11.00 & {$[1-31]$} & 17 & 11.00 & {$[0-31]$} & 18 & 13.00 & {$[6-31]$} \\
\hline CAPE Positive frequency & 35 & 25.00 & {$[17-46]$} & 17 & 24.00 & [18-28] & 18 & 29.50 & {$[21-46]^{*}$} \\
\hline CAPE Positive distress & 35 & 7.00 & {$[1-34]$} & 17 & 6.00 & {$[1-15]$} & 18 & 10.50 & {$[1-34]^{\dagger}$} \\
\hline CAPE Depressive frequency & 35 & 13.00 & {$[6-17]$} & 17 & 13.00 & {$[7-16]$} & 18 & 13.50 & {$[10-17]^{\dagger}$} \\
\hline CAPE Depressive distress & 35 & 8.00 & {$[1-20]$} & 17 & 7.00 & {$[1-19]$} & 18 & 9.00 & {$[2-20]^{\dagger}$} \\
\hline
\end{tabular}

Notes: Comparisons between participants with High and Low Suspiciousness; ${ }^{\dagger} \mathrm{p}<0.10,{ }^{*} \mathrm{p}<0.05,{ }^{* *} \mathrm{p}<0.01$

Data reported as median [range]

alcohol / Cigarette frequency measured as number of days per month alcohol/cigarettes were consumed.

${ }^{\mathrm{b}}$ Alcohol / Cigarette quantity measured as number of standard drinks/cigarettes were consumed per month.

${ }^{\mathrm{S} S P Q}$ dimensions, Cognitive-Perceptual dimension (Ideas of reference + Magical thinking+ Unusual perceptual Experiences+ Suspiciousness); Interpersonal dimensions (Social anxiety + No close friends + Constricted affect); Disorganised dimension (Odd behaviour + Odd speech)

RAVLT, Rey Auditory Verbal Learning Test; BDI, Beck Depression Inventory; STAI-I, State Anxiety Index State score; STAI-II, State Anxiety Index Trait score; SPQ, Schizotypal Personality Questionnaire; CAPE, Community Assessment of Psychic Experiences. 


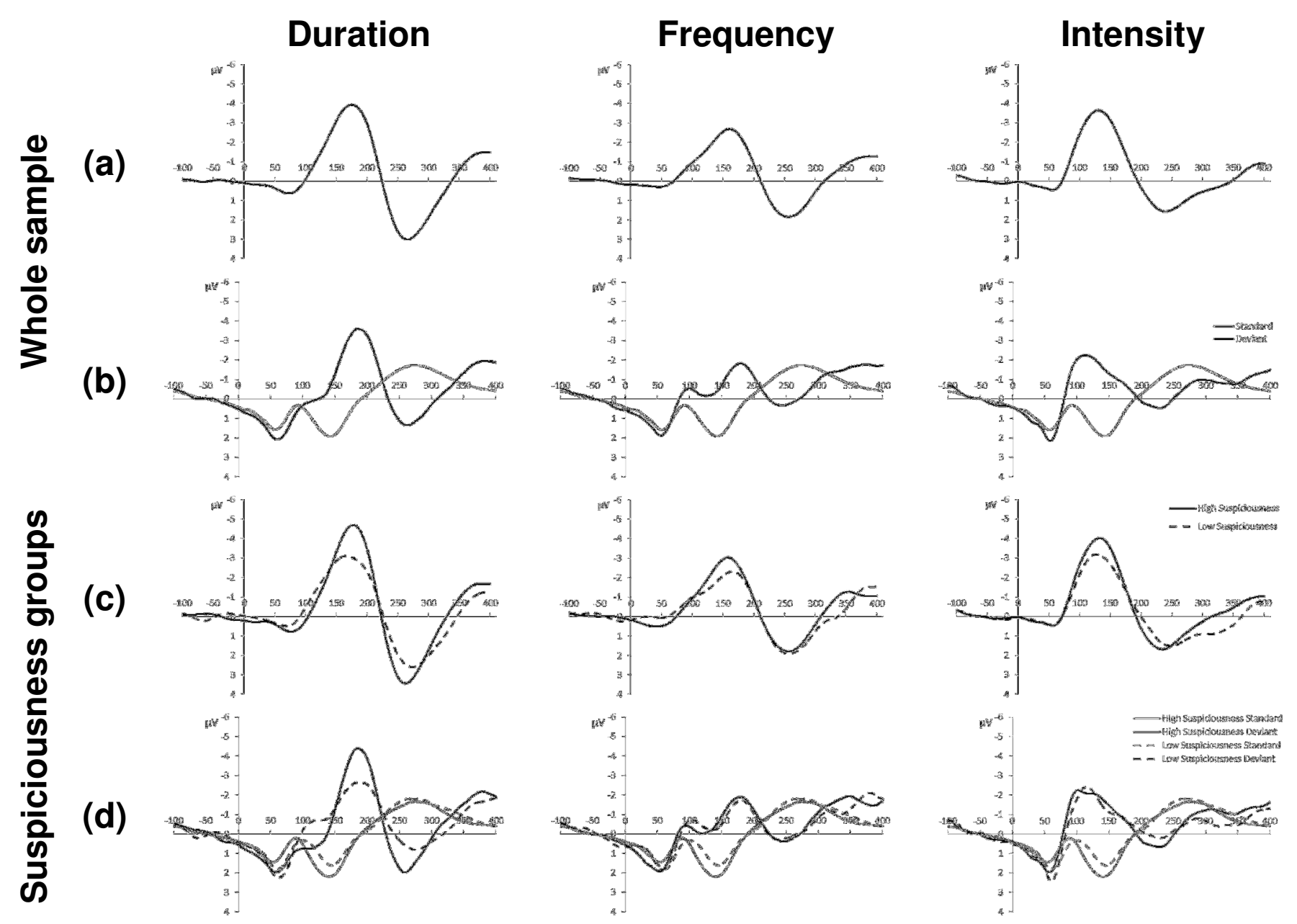

Figure 1.(a) Mismatch negativity (MMN) waveforms at Fz displayed for Duration, Frequency and Intensity deviant conditions. (b) Mastoid reference unsubtracted ERP waveforms at Fz to standard (grey) and deviant tones (black lines). (c) Mismatch negativity (MMN) waveforms at Fz displayed for each condition for the High Suspiciousness group (black solid line) and the Low Suspiciousness group (dashed line). (d) Mastoid reference unsubtracted ERP waveforms at Fz to standard (grey lines) and deviant tones (black lines) for the High Suspiciousness group (solid lines) and for the Low Suspiciousness group (dashed lines). Amplitude is shown in $\mu \mathrm{V}$ on the $\mathrm{y}$-axis and time in milliseconds along the $\mathrm{x}$-axis. 
Duration MMN
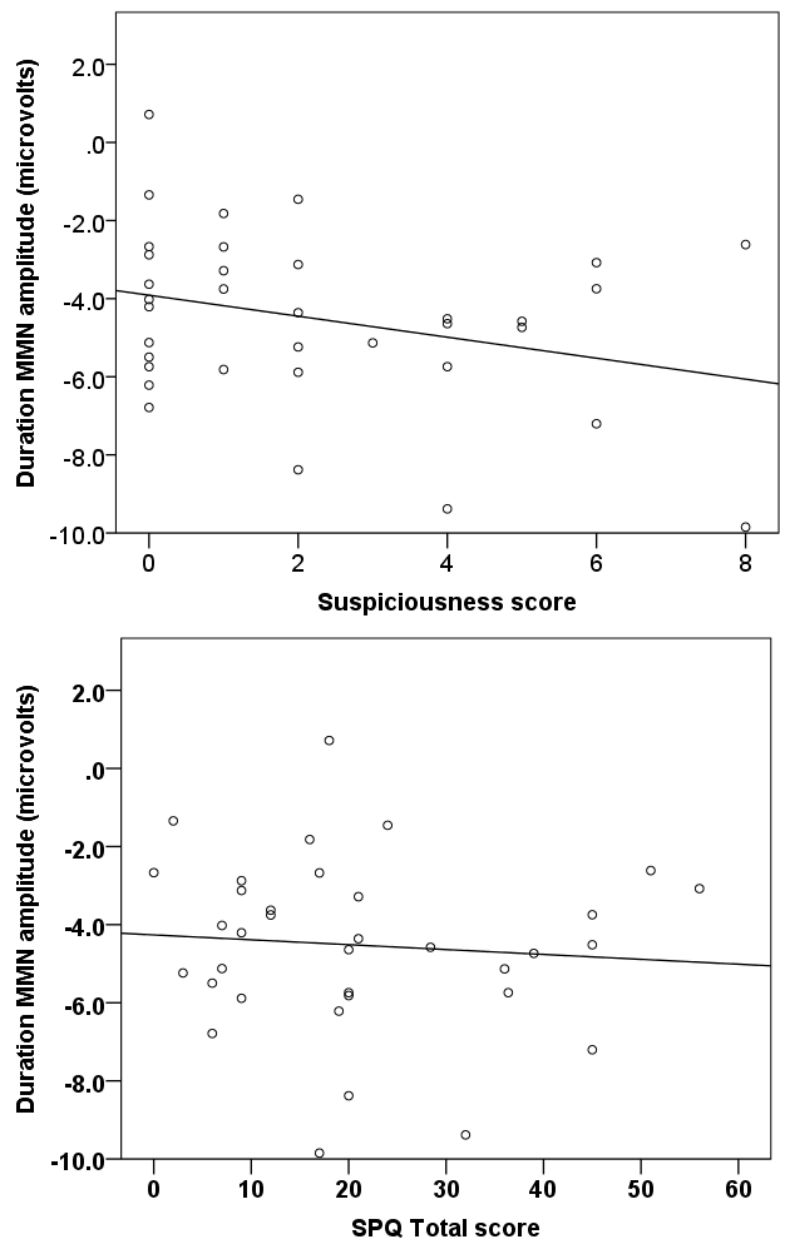

Frequency MMN
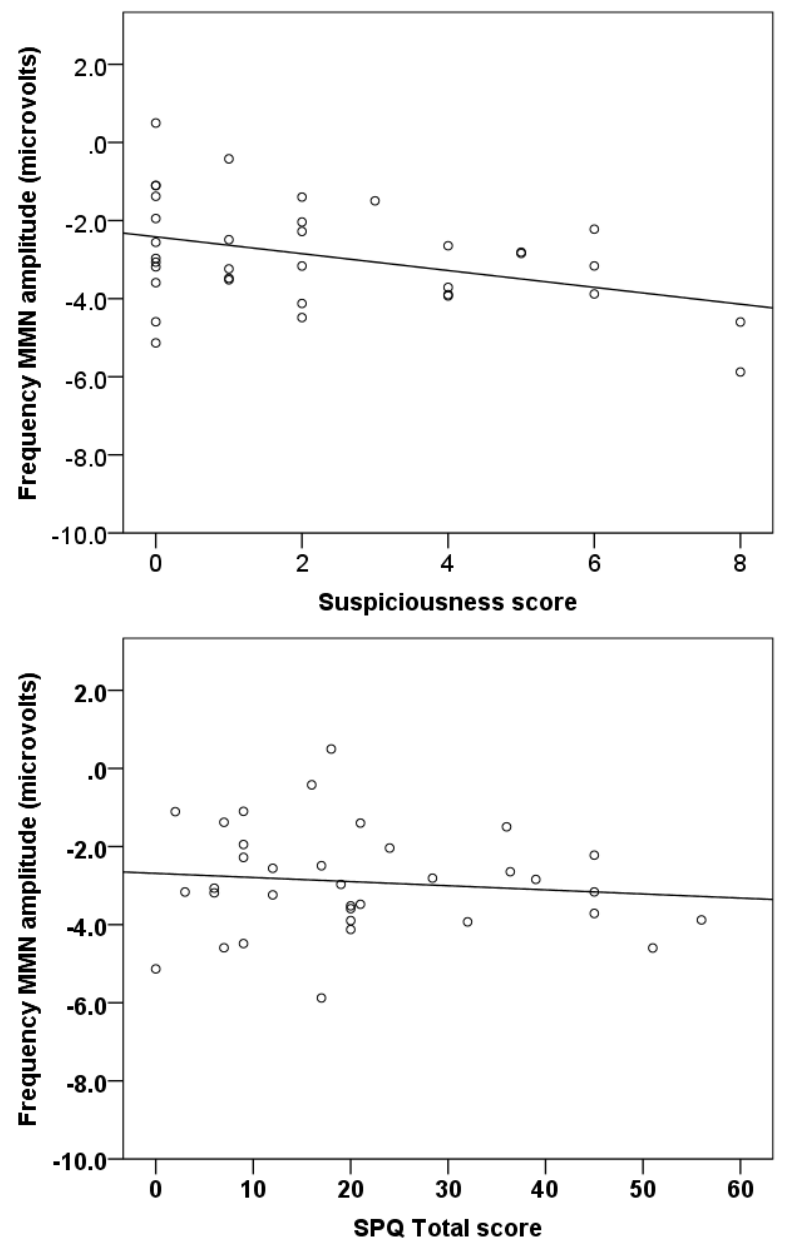

Intensity MMN
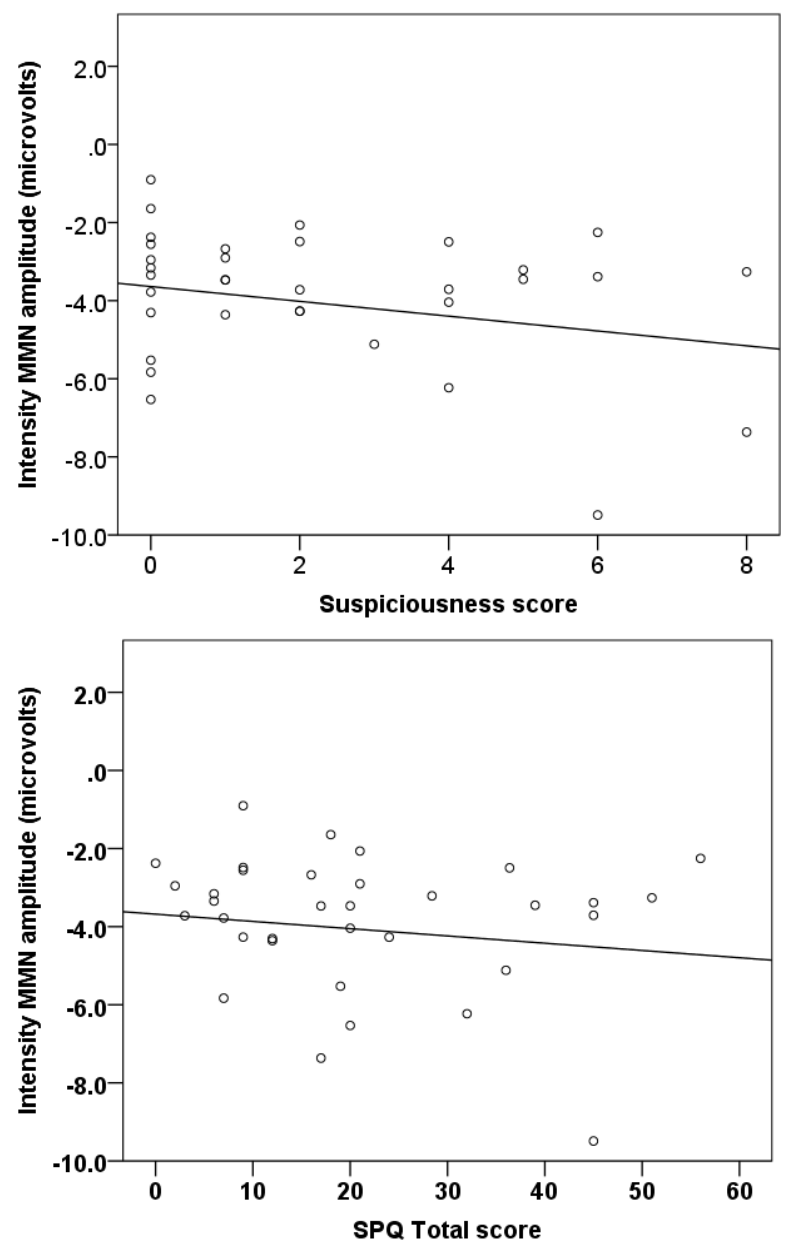

Figure 2. Scatter plots showing association between MMN in the Duration (left), Frequency (middle) and Intensity (right) conditions, Suspiciousness scores (top row) and SPQ total scores (bottom row). 